\title{
In-vitro Cytotoxicity Evaluation of Green Synthesized Gold Nanoparticles and Its Indigenous Mouthwash
}

\author{
Lichi A. Solanki ${ }^{1 *}$ (D) K.K. Shantha Sundari ${ }^{1}$ and S. Rajeshkumar² \\ ${ }^{1}$ Department of Orthodontics, Saveetha Dental College and Hospitals, Saveetha Institute of Medical and \\ Technical Science, Saveetha University, Chennai, India. \\ ${ }^{2}$ Nanobiomedicine Lab, Department of Pharmacology, Saveetha Dental College and Hospitals, Saveetha \\ Institute of Medical and Technical Science, Saveetha University, Chennai, India.
}

\begin{abstract}
The aim of this study was to evaluate the cytotoxicity of the prepared Gold Nanoparticles from Triphala Plant extract and of the indigenous mouthwash prepared from these Triphala based gold nanoparticles (AuNP's) using Artemia salina test. Its objective was to assess whether the prepared mouthwash was safe and biocompatible to use as an alternative to commercial mouthwashes in orthodontic patients to reduce the plaque load around brackets and bands as an adjuvant to tooth brushing. $15 \mathrm{~g}$ of brine shrimps was hatched in salt solution after incubating them for $24 \mathrm{~h}$. Prepared AuNP's at concentrations $0 \mu \mathrm{L}$ (microliter), $5 \mu \mathrm{L}, 10 \mu \mathrm{L}, 20 \mu \mathrm{L}, 30 \mu \mathrm{L}, 50 \mu \mathrm{L}$ were added using a micropipette in one of the microtiter plates and mouthwash prepared from these AuNP's were added at concentrations $0 \mu \mathrm{L}, 10 \mu \mathrm{L}, 20 \mu \mathrm{L}$, $30 \mu \mathrm{L}, 40 \mu \mathrm{L}, 50 \mu \mathrm{L}$ in another microtiter plate. $10 \mathrm{Hatched}$ nauplii (brine shrimps) were added in each of these microtiter plates and incubated for $24 \mathrm{~h}$. All nauplii were alive at the end of $24 \mathrm{~h}$ in the microtiter plate containing AuNP's. At concentrations $0 \mu \mathrm{L}, 10 \mu \mathrm{L}, 20 \mu \mathrm{L}, 30 \mu \mathrm{L}$ nauplii were alive and at $40 \mu \mathrm{L}, 50 \mu \mathrm{L}$ concentrations no nauplii were alive at the end of $24-\mathrm{h}$ in the microtiter plate containing mouthwash. Triphala mediated AuNP's showed no toxic effect on the brine shrimps. AuNP's based mouthwash showed excellent compatibility at lower concentrations. Mild toxic effects were elicited at higher concentrations. Hence, AuNP's could be used to prepare mouthwashes at 20-30 $\mu \mathrm{L}$ concentrations.
\end{abstract}

Keywords: A. nauplii, Brine shrimps, plaque producing organisms

\footnotetext{
*Correspondence: lichisolanki17@gmail.com
}

(Received: June 30, 2020; accepted: April 22, 2021)

Citation: Solanki LA, Sundari KKS, Rajeshkumar S. In-vitro Cytotoxicity Evaluation of Green Synthesized Gold Nanoparticles and Its Indigenous Mouthwash. J Pure Appl Microbiol. 2021;15(2):735-742. doi: 10.22207/JPAM.15.2.25

(C) The Author(s) 2021. Open Access. This article is distributed under the terms of the Creative Commons Attribution 4.0 International License which permits unrestricted use, sharing, distribution, and reproduction in any medium, provided you give appropriate credit to the original author(s) and the source, provide a link to the Creative Commons license, and indicate if changes were made. 


\section{INTRODUCTION}

Nanotechnology is the art of using nanoscience to design nanomaterials and nanoparticles. Nanoparticles (NP's) are particles having size ranging from 1-100nm. Various NP's that are being experimented in the field of medicine are gold, silver, ruthenium, iridium, palladium etc. Of all, gold nanoparticles (AuNP's) have superior properties as gold is inert, have better compatibility with animal and plant cells and is nontoxic ${ }^{1}$.

In recent times, NP's have gained popularity in the field of dentistry as well ${ }^{2}$. NP's have been used for various applications like site specific drug delivery, as sensors, anticancer drugs, antioxidants, larvicides, Nanofluids, antimicrobial agents in dentistry ${ }^{3}$, as mouthwashes ${ }^{4}$ and for treatment of white spot lesions during orthodontic treatment ${ }^{2}$, etc. Various physical and chemical methods used to produce NPs are expensive. Toxic reducing and capping agents are employed in these methods. Therefore, green synthesis of NP's has gained ground owing to its cost effectiveness and environmental compatibility 5 . NP's carrying drugs have extended half-life period, longer circulation time, and carry higher concentration of drugs ${ }^{6}$. Unlike Bulk metals, NP's possess good optical properties as the motions of electrons are restrained owing to the reduced size. Due to the ultra-small size, they are less toxic and have a high surface to volume ratio. Hence, interactions take place at the surface resulting in faster absorption ${ }^{7}$ and relatively high the drug loading ${ }^{6}$. The large surface area, high charge density of NPs enables them to interact with the negatively-charged surface of bacterial cells to a greater extent resulting in enhanced antimicrobial activity ${ }^{8}$.

NP's influence biological systems in multiple ways. Size of the NP's determine their cellular uptake, endocytosis, cytotoxicity, bio distribution, and clearance pathway. Bio-nano interface reactions affect the size and surface properties of the NPs after binding with proteins, glycans, ions and hence ${ }^{9}$ alter the uptake pathways (nonspecific and receptor-mediated). Conversely, bio-nano interactions may cause some change in the protein composition, the distribution of the biological molecules in the organism, and may constitute or show some hidden immune epitopes.
Improved understanding of nanotoxicity will help to avoid its unfavourable effects ${ }^{10}$.

In this in-vitro study, brine shrimp assay was used to evaluate the toxicity of prepared AuNP's from triphala extract. It also studied the cytotoxicity of the mouthwash prepared from it. Brine shrimp assay was first proposed by Michael et al. 1956, then developed by Vanhaecke et al. 1981. It has had its applications in detecting fungal toxins, heavy metals, plant extract toxicity, cytotoxicity testing of dental materials ${ }^{11}$. Artemia (A.nauplii) is a genus of aquatic crustaceans, a zooplankton, known as brine shrimps. These species have small size, short life span, large offspring production, high adaptability to hypersaline environments at various temperatures. Various nanotoxicology conducted on animals proved to be time consuming due to the tedious procedure of obtaining ethical clearance. To overcome this, preference is given to in-vitro studies for evaluating toxicity. Hence, A.nauplii have been used as invertebrate models in nanotoxicology $y^{1,2,3}$.

In this present study, the green synthesis method was employed to fabricate AuNP's using Triphala extract (Terminalia chebula, Terminalia bellerica, and Embelica officinalis). AuNP's were characterized by UV-Vis spectroscopic method which showed SPR (surface plasmon resonance peak) at $520 \mathrm{~nm}$, TEM (Transmission electron Microscope) analysis which revealed the size of AuNP's to be $12-35 \mathrm{~nm}$, spherical in shape. Mouthwash was prepared from these Triphala mediated AuNP's and antimicrobial effect of these AuNP's and its based mouthwash was evaluated against most commonly found oral pathogens in oral cavity and in orthodontic patients. Antimicrobial effect was tested against Enterococcus faecalis, Streptococcus mutans, Staphylococcus aureus and Candida Albicans at various concentrations. These AUNP's and its mouthwash showed good antibacterial activity against these pathogens. For this reason, prior to use in orthodontic patient's biocompatibility was evaluated using brine shrimp assay.

According to a study done by Anderson, it has been proved that chlorhexidine is effective in maintaining orthodontic hygiene due to its excellent antibacterial activity, thus causing a reduction in the gingival problems. But in the same 
study, they mentioned shortcomings like burning sensation, staining, discolouration of tongue and composite restorations ${ }^{13}$. Another research by Balalakshmi et al stated that it is a contraindicated to use it in patients who are immunocompromised and have mucositis as there is presence of alcohol in $0.2 \%$ chlorhexidine mouthwashes ${ }^{3}$.

NP based mouthwash has been known to overcome certain disadvantages of commercial conventional mouthwashes. In a study by Norouzzadeh Helali et al metal oxide nanoparticles like $\mathrm{Ag} / \mathrm{ZnO}$ or $\mathrm{ZnO}$ were checked for the antimicrobial activity against S. mutans and compared with $0.2 \%$ chlorhexidine mouthwash. The cytotoxicity of the $\mathrm{ZnO}$ and $\mathrm{Ag} /$ $\mathrm{ZnO}$ nanoparticles was evaluated in the A549 cell line. The results proved that no significant ill-effect is caused to the cells up to $0.1 \mathrm{mg} / \mathrm{ml}$ of $\mathrm{ZnO}$ and $\mathrm{Ag} / \mathrm{ZnO} N \mathrm{NP}^{\prime} \mathrm{s}$. It was concluded that $\mathrm{Ag} / \mathrm{ZnO} \mathrm{NP}$ based mouthwash is an effective antimicrobial agent against $S$. mutans and could be considered as an alternative to $0.2 \%$ chlorhexidine $^{14}$.

Thus, the objective of this study was to evaluate the biocompatibility of the prepared AuNP's and the mouthwash which could be used as an alternative to commercially available mouthwashes.

\section{MATERIALS AND METHODS}

Synthesis and antimicrobial evaluation of AuNP's and its based mouthwash

Owing to the good antimicrobial property possessed by Triphala plant extract and gold nanoparticles, the combination was selected to produce AuNP's and AuNP based mouthwash. Triphala extract powder was crushed into coarse powder and mixed with Gold chloride solution. The mixture was heated at 50 degrees and was homogenously mixed on an orbital shaker. The colour was pale yellow and it turned purple, this indicated the formation of AuNP's. This formation of AuNp's was confirmed using spectroscopic analysis which showed a peak of $520 \mathrm{~nm}$ corresponding to the surface plasmon resonance of AuNPs. Characterization using Transmission Electron Microscopy revealed spherical AuNP's with a size in the range of $18-35 \mathrm{~nm}$. The antimicrobial property for AuNP's was then assessed using Zone of inhibition test. C.albicans exhibited excellent antimicrobial activity followed by S. mutans.
S. aureus and E. coli also showed intermediate antimicrobial potential. AuNP based mouthwash was prepared using $600 \mu \mathrm{L}$ AuNP's and all basic ingredients of mouthwash. The mouthwash also depicted good antimicrobial activity against all pathogens.

Chemicals used: 1) AuNP's prepared from triphala plant extract. 2) mouthwash prepared from triphala mediated AuNP's. 3) A. nauplii eggs. Hatching the brine shrimp

$27 \mathrm{~g}$ of table salt was weighed and added to 3 litres of distilled water in a cylindrical jar and thoroughly stirred. For good aeration, an air pump was placed into the bottom of the jar. $15 \mathrm{~g}$ of brine shrimp eggs were added at the top-level water of the jar and mixed. A light bulb (60-100-Watt bulb) was placed a few inches from the jar. After 20-24 h of incubation period, nauplii hatched. Hatched nauplii was separated from the empty egg by turning off air and switching off the lamp. This ensured that the empty eggs were floating on top and the brine shrimp were concentrated at the bottom of the water column.

\section{Microscopic evaluation of the brine shrimps}

Two brine shrimp eggs were inoculated on a clean slide with 2 drops of distilled water. They were observed under a light microscope at 40X and 10X magnification. (Fig. 3) Shrimps had an unsegmented body and a single eye, about 22 $\mathrm{mm}$ long.

Toxicity testing on the Artemia nauplii: (Fig. 2)

At the end of $24 \mathrm{~h}$, wells of the microtiter plates were inoculated with AuNP's of $0 \mu \mathrm{L}$, $5 \mu \mathrm{L}, 10 \mu \mathrm{L}, 20 \mu \mathrm{L}, 30 \mu \mathrm{L}, 50 \mu \mathrm{L}$ concentrations. Mouthwash at concentrations $0 \mu \mathrm{L}, 10 \mu \mathrm{L}, 20 \mu \mathrm{L}$, $30 \mu \mathrm{L}, 40 \mu \mathrm{L}, 50 \mu \mathrm{L}$ was added in another microtiter plate. In one of the wells in both plates, a sterile salt solution was used as control. $A$. nauplii were collected in a petri dish from the cylindrical jar. 10 hatched nauplii were collected using a dropper and added in each well in both plates. They were counted using a magnifying glass. This was cross checked by another researcher to avoid any error. At the end of $24 \mathrm{~h}$, nauplii in each well in each of the microtiter plates were counted.

\section{RESULTS AND DISCUSSION}

At the end of $24 \mathrm{~h}$, the microtiter plate which was inoculated with AuNP's alone showed all $A$. nauplii to be alive in each well and in the 
well with the control group. In the microtiter plate containing mouthwash 10 nauplii were alive at $0 \mu \mathrm{L}, 8$ nauplii at $10 \mu \mathrm{L}, 7$ nauplii at $20 \mu \mathrm{L}, 3$ nauplii at $30 \mu \mathrm{L}$, Onauplii at $40 \mu \mathrm{L}$ and $50 \mu \mathrm{L}$.

The cytotoxic effect of AuNP's on $A$. nauplii revealed that all brine shrimps are alive at the end of $24 \mathrm{~h}$ which indicate no cytotoxic effect of AuNP's on the brine shrimps. The cytotoxic
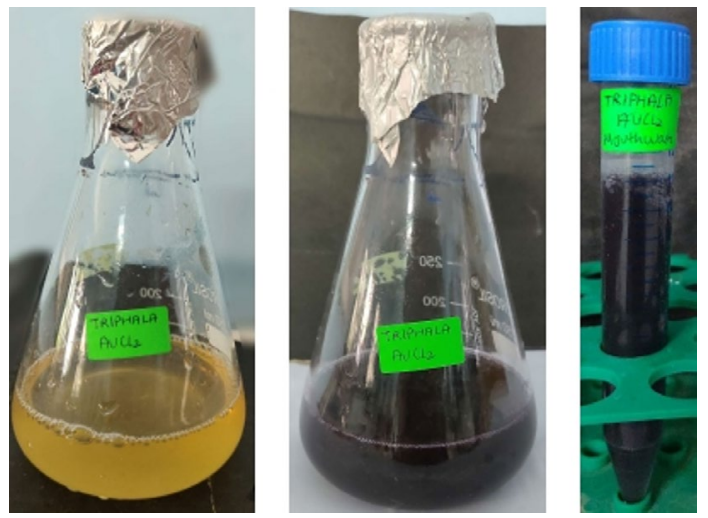

Fig. 1. Triphala plant mediated AuNP's and its based mouthwash.

It represents color change from pale yellow to violet indicating formation of Gold nanoparticles from which the mouthwash is prepared. effect of AuNP based indigenous mouthwash on A. nauplii revealed that all brine shrimps are alive at the end of $24 \mathrm{~h}$ at low concentrations which indicate no cytotoxic effect of AuNP's on the brine shrimps. At higher concentrations, the brine shrimps are not alive indicating the toxic effect of the mouthwash on brine shrimps at higher concentrations.

\section{Mortality rate was calculated as follows}

$$
\begin{gathered}
\text { Mortality }(\%)=(\text { Number of dead nauplii / Total } \\
\text { number of A. nauplii } \times 100
\end{gathered}
$$

Mortality rate for AuNP's was $0 \%$ and for AuNP based mouthwash was $46.6 \%$.

In this current study, triphala plant extract mediated AuNP's and mouthwash (Figure 1) showed good antimicrobial activity against Enterococcus faecalis, Streptococcus mutans, Staphylococcus aureus and Candida Albicans. Therefore, toxicity of AuNP's was evaluated to so AuNP's could be used for oral applications for bacterial load reduction. NP based mouthwashes do not possess the disadvantages of commercially used ones. They have been known to have
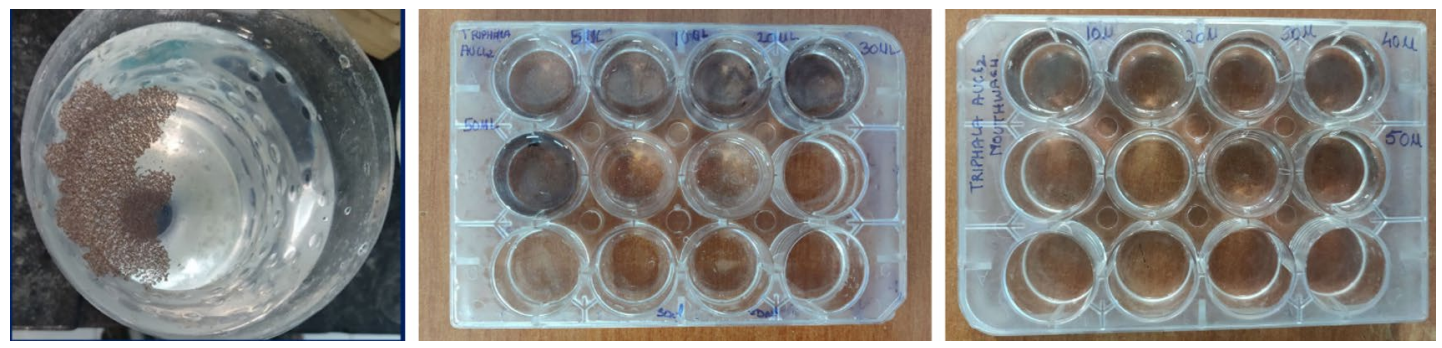

Fig. 2. Hatched brine shrimps

(left), AuNP's with A.nauplii (centre), Mouthwash with A.nauplii (right).
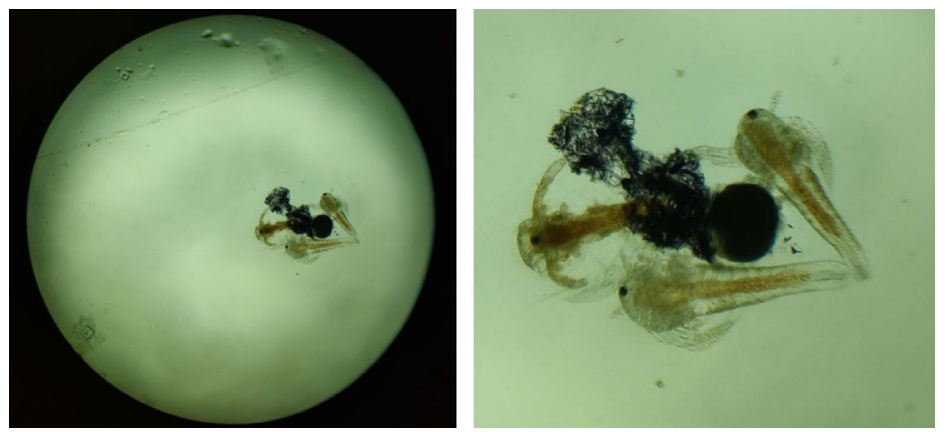

Fig. 3. Light microscope - A.nauplii : 40X (left) , 10X (right); represents the microscopic image of the brine shrimp attached to a gold nanoparticle. 
comparable antimicrobial activity as the gold standard mouthwashes. In an attempt to use it as an adjunct to mechanical cleansing in orthodontic patients for reduction of plaque load and white spot lesions, its cytotoxic evaluation was carried out on brine shrimps ${ }^{14}$.

The oral cavity encounters a plethora of pathogens. Plaque biofilm- a major cause of caries, periodontitis and other dental diseases- is a complex community of various microorganisms that cause infections ${ }^{15}$. Many methods have been employed but the desired outcome is never achieved due to fast degradation and rapid release of antimicrobial agents resulting in low efficiency and safety concerns ${ }^{16,17}$. NPs are tiny particles with a diameter of 1-100 $\mathrm{nm}$. They are useful in antibacterial therapies because of improved characteristics like ultra-small sizes, large surface-area-to-mass ratio and increased chemical reactivity ${ }^{16}$. NPs may be able to come up with a new strategy for treatment of and prevention of dental infections ${ }^{18}$. The large surface area and high charge density of NPs help them to come in contact with the negatively-charged surface of bacterial cells to a greater extent resulting in enhanced antibacterial activity ${ }^{8}$. Metal and organic NPs have been of use in dentistry due to their broad-spectrum antimicrobial properties ${ }^{18}$.

Certain studies have proven that NP's are nontoxic within certain concentration ranges $^{19-25}$. Some other studies show that the low concentrations are nontoxic but larger concentrations may have some toxic effect. A few other researchers conclude that toxicity of NP's is dose dependent ${ }^{26-28}$. In conclusion, the toxicity of antimicrobial NPs is caused by multiple factors like dosage, types, distribution, duration of action, particle size, interaction with other compounds ${ }^{2}$. Nanoparticles require special attention, as these compounds in this ultra-small size range have chemical properties that vary in comparison with those of their larger counterparts.

Artemia is one of the most valuable test crustaceans used for marine ecotoxicity testing as it is widely used as a nutritious live food source to the larvae of a variety of marine organisms. This makes them the most convenient, least labourintensive live food available for aquaculture and will be impacted greatly by the release of NP 's into aquatic systems because of their greater interaction with the environment via nonselective filter feeding ${ }^{29}$.

Initially, in-vivo animal studies were profoundly used for assessing toxicity. But animal rights activists from IACUC criticized this idea. Due to high costs and increased time in in-vivo studies, certain in - vitro methods were employed. These techniques were XTT assay, MTT assay, cell culture, the WST-1 assay, BrdU assay, fluorescence Microscopy and $\mathrm{LDH}^{11}$. But these techniques were time consuming and expensive. In an attempt to obtain a rapid and cost-effective screening technique brine shrimp assay test was introduced and is being currently used in assessing the toxicity of nanoparticles ${ }^{30}$. Many studies have used brine shrimps for evaluating biocompatibility ${ }^{31-35}$.

Our study concludes that the AuNP's have no toxic effect on the brine shrimps. AuNP's based mouthwash showed a very mild toxic effect as the concentrations were raised to $40 \mu \mathrm{L}$ and $50 \mu \mathrm{L}$. Gold nanoparticle-based mouthwash can be made using low concentrations of NP's.

Silver nanoparticles and $\mathrm{ZnO}$ based mouthwashes have been shown to have comparable antimicrobial properties like the most commonly used mouthwash in dentistry i.e., Chlorhexidine. Silver nanoparticle and $\mathrm{ZnO}$ based mouthwashes have been used effectively in Orthodontic patients ${ }^{29,14}$. Along with good antimicrobial properties, silver nanoparticles have been known to have cytotoxic effect of human dermal fibroblasts at high concentrations ${ }^{26}$. Sreenivasagan et al proved its cytotoxicity at 20 and $25 \mu \mathrm{L}^{36}$. A study reported by Cierech et concluded cytotoxic effect of Zinc oxide nanoparticles beyond a concentration of $20 \mu \mathrm{L}^{37}$. No literature is available on gold nanoparticle-based mouthwashes. Further studies need to be carried out on use of gold nanoparticle-based mouthwashes. There is a dire need that this Triphala plant mediated AuNP based mouthwash needs to be compared with other commercially available mouthwashes to evaluate its antimicrobial properties.

\section{Limitations}

There is no study reported on gold nanoparticle-based mouthwashes in dental patients. Therefore, more studies are needed in this field. AuNP based mouthwash needs to be compared with other nanoparticle-based mouthwashes and with other commercially 
available mouthwashes. Antimicrobial activity against most commonly found Lactobacillus spp in orthodontic patients was not included in this study. Hence, this must be tested too. Cost of gold nanoparticles needs to be taken into account to make it affordable. Cytotoxicity testing was done at aquatic level. Further toxicity tests should be employed prior to employing its use in orthodontic patients.

\section{CONCLUSION}

This study has shown that AuNP's have no toxic effect on the brine shrimps upto 20 $\mu \mathrm{L}$ concentration. There is a mild toxic effect elicited by the mouthwash at $30 \mu \mathrm{L}$ and toxixcity increased as the concentration of the mouthwash is increased. Hence, it is a potential alternative to commercially available mouthwashes in orthodontic patients at 20-30 $\mu \mathrm{L}$ concentration. AuNP based mouthwashes could be used as an adjuvant to mechanical cleansing like tooth brushing to reduce the bacterial load after testing it at higher levels. As other nanoparticles have reported harmful effects on the oral cavity with increasing concentrations, AuNp's should be studied more elaborately owing to their unique properties.

\section{ABBREVIATIONS}

AuNP's : Gold Nanoparticles; NPs : Nanoparticles; $\mu \mathrm{L}$ : Microliter; ZnO: Zinc oxide; IACUC: Institutional Animal Care and Use Committees.

\section{ACKNOWLEDGMENTS}

I would like to acknowledge the Head of the Department of Orthodontics and Dentofacial Orthopaedics, Dr. Aravind Kumar Subramaniyan for his constant support and encouragement.

\section{CONFLICT OF INTEREST} conflict of interest.

The authors declare that there is no

\section{AUTHOR'S CONTRIBUTIONS}

All authors designed the experiment. LS and RK performed the experiments. LS, RK, SK analysed the data. LS wrote the manuscript. All the authors read and approved the manuscript.

\section{FUNDING}

None.

\section{DATA AVAILABILITY}

All the datasets generated or analysed during this study are included in the manuscript.

\section{ETHICS STATEMENT}

Not applicable.

\section{REFERENCES}

1. Umamaheswari C, Lakshmanan A, Nagarajan NS. Green synthesis, characterization and catalytic degradation studies of gold nanoparticles against congo red and methyl orange. J Photochem Photobiol B. 2018;178:3339.doi: 10.1016/j.jphotobiol.2017.10.017

2. Song W, GeS. Application of Antimicrobial Nanoparticles in Dentistry. Molecules. 2019;24(6):1033. doi: 10.3390/molecules24061033

3. Balalakshmi C, Gopinath K, Govindarajan M, et al. Green synthesis of gold nanoparticles using a cheap Sphaeranthus indicus extract: Impact on plant cells and the aquatic crustacean Artemia nauplii. J Photochem Photobiol B. 2017;173:598-605. doi: 10.1016/j. jphotobiol.2017.06.040

4. Abadi MFD, Mehrabian S, Asghari B, Namvar AE, Ezzatifar F, Lari AR. Silver nanoparticles as active ingredient used for alcohol-free mouthwash. GMS Hyg Infect Control. 2013;8(1):Doc05. doi: 10.3205/ dgkh000205

5. Zha J, Dong C, Wang X, Zhang X, Xiao X, Yang X. Green synthesis and characterization of monodisperse gold nanoparticles using Ginkgo Biloba leaf extract. Optik. 2017;144:511-521. doi:10.1016/j.ijleo.2017.06.088.

6. Balakrishnan G, Shil S, Vijalakashmi N, Rao MR, Prabhu K. Green synthesis of copper nanocrystallites using triphala churna and their anti-microbial studies. Drug Invention Today. 2019;12(9):2038-2044.

7. Chen H, Zhou K, Zhao G. Gold nanoparticles: From synthesis, properties to their potential application as colorimetric sensors in food safety screening. Trends Food Sci Technol. 2018;78:83-94. doi: 10.1016/j. tifs.2018.05.027

8. Cao W, Zhang Y, Wang $X$, et al. Novel resin-based dental material with anti-biofilm activity and improved mechanical property by incorporating hydrophilic cationic copolymer functionalized nanodiamond. J Mater Sci Mater Med. 2018;29(10):162. doi: 10.1007/ s10856-018-6172-z

9. Pan Y, Bartneck M, Jahnen-Dechent W. Cytotoxicity of Gold Nanoparticles. Methods in Enzymology. 2012;509:225-242. doi: 10.1016/b978-0-12-3918581.00012-5

10. Thota S, Crans DC. Metal Nanoparticles: Synthesis and Applications in Pharmaceutical Sciences. John Wiley \& Sons; 2018. doi: 10.1002/9783527807093

11. Barahona MV, Sánchez-Fortún S. Toxicity of carbamates to the brine shrimp Artemia salina and the effect of 
atropine, BW284c51, iso-OMPA and 2-PAM on carbaryl toxicity. Environ Pollut. 1999;104(3):469-476. doi: 10.1016/S0269-7491(98)00152-3

12. Muhammad W, Ullah N, Khan M, Ahmad W, Khan MQ Abbasi BH. Why Brine shrimp (Artemia salina) larvae is used as system for nanomaterials? The science of procedure and nano-toxicology: A review. Int. J. Biosci. 2019;14(5):156-176. doi: 10.12692/ijb/14.5.156176

13. Anderson GB, Bowden J, Morrison EC, Caffesse RG. Clinical effects of chlorhexidine mouthwashes on patients undergoing orthodontic treatment. American Journal of Orthodontics and Dentofacial Orthopedics. 1997;111(6):606-612. doi:10.1016/ s0889-5406(97)70312-3

14. Kachoei M, Divband B, Dabaghi Tabriz F, Norouzzadeh Helali Z, Esmailzadeh M. A comparative study of antibacterial effects of mouthwashes containing $\mathrm{Ag}$ / $\mathrm{ZnO}$ or $\mathrm{ZnO}$ nanoparticles with chlorhexidine and investigation of their cytotoxicity. Nanomedicine Journal. 2018;5(2):102-110. doi: 10.22038/ nmj.2018.005.007

15. Kasraei S, Sami L, Hendi S, Alikhani M-Y, Rezaei-Soufi L, Khamverdi Z. Antibacterial properties of composite resins incorporating silver and zinc oxide nanoparticles on Streptococcus mutans and Lactobacillus. Crestor Dent Endod. 2014;39(2):109-114. doi: 10.5395/ rde.2014.39.2.109

16. Saafan A, Zaazou MH, Sallam MK, Mosallam O, El Danaf HA. Assessment of Photodynamic Therapy and Nanoparticles Effects on Caries Models. Open Access Maced J Med Sci. 2018;6(7):1289-1295. doi: 10.3889/ oamjms.2018.241

17. Cao W, Zhang $Y$, Wang $X$, et al. Development of a novel resin-based dental material with dual biocidal modes and sustained release of $\mathrm{Ag}+$ ions based on photocurable core-shell $\mathrm{AgBr} /$ cationic polymer nanocomposites. J Mater Sci Mater Med. 2017;28(7):103. doi: 10.1007/s10856-017-5918-3

18. Magalhaes APR, Moreira FCL, Alves DRS, et al. Silver nanoparticles in resin luting cements: Antibacterial and physiochemical properties. Journal of Clinical and Experimental Dentistry. 2016:8(4):e415-422. doi:10.4317/jced.52983

19. Sabatini C, Mennito AS, Wolf BJ, Pashley DH, Renné WG. Incorporation of bactericidal poly-acrylic acid modified copper iodide particles into adhesive resins. J Dent. 2015;43(5):546-555. doi: 10.1016/j. jdent.2015.02.012

20. Monzavi A, Eshraghi S, Hashemian R, Momen-Heravi F. In vitro and ex vivo antimicrobial efficacy of nano-MgO in the elimination of endodontic pathogens. Clin Oral Investig. 2015;19(2):349-356. doi: 10.1007/s00784014-1253-y

21. Li P, Tong Z, Huo L, Yang F, Su W. Antibacterial and biological properties of biofunctionalized nanocomposites on titanium for implant application. J Biomater Appl. 2016;31(2):205-214. doi: 10.1177/0885328216645951

22. Liu X, Gan K, Liu H, Song X, Chen T, Liu C. Antibacterial properties of nano-silver coated PEEK prepared through magnetron sputtering.
Dent Mater. 2017;33(9):e348-e360. doi: 10.1016/j. dental.2017.06.014

23. Zhu Y, Cao H, Qiao S, et al. Hierarchical micro/ nanostructured titanium with balanced actions to bacterial and mammalian cells for dental implants. Int J Nanomedicine. 2015;10:6659-6674. doi: 10.2147/IJN. S92110

24. Yang T, Qian S, Qiao Y, Liu X. Cytocompatibility and antibacterial activity of titania nanotubes incorporated with gold nanoparticles. Colloids Surf B Biointerfaces. 2016;145:597-606. doi: 10.1016/j. colsurfb.2016.05.073

25. Sodagar A, Bahador A, Pourhajibagher M, Ahmadi $B$, Baghaeian P. Effect of Addition of Curcumin Nanoparticles on Antimicrobial Property and Shear Bond Strength of Orthodontic Composite to Bovine Enamel. J Dent, 2016;13(5):373-382.

26. Pérez-Díaz MA, Boegli $L$, James $G$, et al. Silver nanoparticles with antimicrobial activities against Streptococcus mutans and their cytotoxic effect. Mater Sci Eng C. 2015;55:360-366. doi: 10.1016/j. msec.2015.05.036

27. Cao H. Silver Nanoparticles for Antibacterial Devices: Biocompatibility and Toxicity. CRC Press; New York; 2017. doi: $10.1201 / 9781315370569$

28. Niska K, Knap N, Kędzia A, Jaskiewicz M, Kamysz W, Inkielewicz-Stepniak I. Capping Agent-Dependent Toxicity and Antimicrobial Activity of Silver Nanoparticles: An In Vitro Study. Concerns about Potential Application in Dental Practice. International Journal of Medical Sciences. 2016;13(10):772-782. doi:10.7150/ijms.16011

29. Arulvasu C, Jennifer SM, Prabhu D, Chandhirasekar D. Toxicity Effect of Silver Nanoparticles in Brine Shrimp Artemia. The Scientific World Journal. 2014;2014:1-10. doi:10.1155/2014/256919

30. Michael AS, Thompson CG, Abramovitz M. Artemia salina as a Test Organism for Bioassay. Science. 1956;123(3194):464. doi: 10.1126/ science.123.3194.464

31. Apu AS, Muhit MA, Tareq SM, Pathan AH, Jamaluddin ATM, Ahmed M. Antimicrobial Activity and Brine Shrimp Lethality Bioassay of the Leaves Extract of Dillenia indica Linn. Journal of Young Pharmacists. 2010;2(1):50-53. doi:10.4103/0975-1483.62213

32. Meyer B, Ferrigni N, Putnam J, Jacobsen L, Nichols D, McLaughlin J. Brine Shrimp: A Convenient General Bioassay for Active Plant Constituents. Planta Medica. 1982;45(05):31-34. doi:10.1055/s-2007-971236.

33. Solis P, Wright C, Anderson M, Gupta M, Phillipson J. A Microwell Cytotoxicity Assay using Artemia salina (Brine Shrimp). Planta Medica. 1993;59(03):250-252. doi:10.1055/s-2006-959661

34. Supraja N, Prasad TNVKV, Gandhi AD, Anbumani $D$, Kavitha P, Babujanarthanam R. Synthesis, characterization and evaluation of antimicrobial efficacy and brine shrimp lethality assay of Alstonia scholaris stem bark extract mediated ZnONPs. Biochem Biophys Rep. 2018;14:69-77. doi: 10.1016/j. bbrep.2018.04.004

35. Sreekanth TVM, Nagajyothi PC, Supraja N, T N $\checkmark \mathrm{K}$. Evaluation of the antimicrobial activity and 
cytotoxicity of phytogenic gold nanoparticles. Applied Nanoscience. 2015;5(5):595-602. doi:10.1007/s13204014-0354-x

36. Sreenivasagan $S$, Subramanian AK, Shanmugam R. "Assessment of antimicrobial activity and cytotoxic effect of green mediated silver nanoparticles and its coating onto mini-implants." Annals of Phytomedicine,
2020;9(1): 207-212. doi: 10.21276/ap.2020.9.1.27

37. Cierech M, Wojnarowicz J, Kolenda A, et al. Zinc Oxide Nanoparticles Cytotoxicity and Release from Newly Formed PMMA-ZnO Nanocomposites Designed for Denture Bases. Nanomaterials. 2019; 9(9):1318. doi: 10.3390/nano9091318. 